June 16, 2004

\title{
Coherent Switching with Decay of Mixing: An Improved Treatment of Electronic Coherence for non-Born-Oppenheimer Trajectories
}

\author{
Chaoyuan Zhu, Shikha Nangia, Ahren W. Jasper, and Donald G. Truhlar \\ Department of Chemistry and Supercomputing Institute, \\ 207 Pleasant Street S.E., University of Minnesota, \\ Minneapolis, MN 55455-0431
}

\begin{abstract}
The self-consistent decay of mixing (SCDM) semiclassical trajectory method for electronically nonadiabatic dynamics is improved by modifying the switching probability that determines the instantaneous electronic state toward which the system decoheres. The new method is called coherent switching with decay of mixing (CSDM), and it differs from the previously presented SCDM method in that the electronic amplitudes controlling the switching of the decoherent state are treated fully coherently in the electronic equations of motion for each complete passage through a strong interaction region. The new method is tested against accurate quantum mechanical calculations for twelve atom-diatom scattering test cases. Also tested are the SCDM method and the trajectory surface hopping method of Parlant and Gislason that requires coherent passages through each strong interaction region, and which we call the ECP-TSH method. The results are compared with previously presented results for the fewest-switches with time uncertainty and Tully's fewest switches (TFS) surface hopping methods and the semiclassical Ehrenfest method. We find that the CSDM method is the most accurate of the semiclassical trajectory methods tested. Including coherent passages improves the accuracy of the SCDM method (i.e., the CSDM method is more accurate than the SCDM method) but not of the trajectory surface hopping method (i.e., the ECP-TSH method is not more accurate on average than the TFS method).
\end{abstract}




\section{INTRODUCTION}

The development of semiclassical methods for non-Born-Oppenheimer trajectories (i.e., classical or quasiclassical molecular dynamics that involves coupled electronic states) requires a delicate blending of quantum mechanics for the electronic motions with classical (or quasiclassical) trajectories for the nuclear motions. ${ }^{1}$ The treatment of Born-Oppenheimer breakdown, therefore, requires an artful approach, ${ }^{2}$ and several methods have been developed. We distinguish four general classes of methods, which differ in their treatment of electronic decoherence. Decoherence is defined in this article as the tendency of the timeevolved density matrix to assume a form corresponding to a statistical ensemble of states rather than a coherent combination of state wave vectors. This is sometimes called dephasing or disentanglement.

The first general approach to non-Born-Oppenheimer trajectories is the trajectory surface hopping ${ }^{1}(\mathrm{TSH})$ approach, in which individual trajectories evolve independently on a single potential energy surface with occasional instantaneous hops or switches between surfaces. The most successful of the well-tested versions of this approach are Tully's fewest switches (TFS) method $^{3}$ and the fewest switches with time uncertainty (FSTU) method. ${ }^{4,5}$ For these methods, one can think of an accurate time-dependent wave packet as being modeled by a swarm of independent trajectories propagating on the different electronic surfaces. Each trajectory hops stochastically between the electronic surfaces according to an associated electronic density matrix, different for each trajectory, which is obtained by propagating the solution to the time-dependent electronic Schrödinger equation coherently along the classical trajectory. The electronic density matrices for any two trajectories in the ensemble may differ for two reasons: (1) the two trajectories have different initial 
coordinates and/or momenta, and (2) they hop at different times along their trajectories. The ensemble averaged electronic density matrix (which may be compared with the accurate quantum mechanical electronic density matrix) effectively decoheres (i.e., the off-diagonal elements systematically tend toward zero) for the two reasons mentioned above, i.e., due to the initial width of the wave packet and due to the divergence of trajectories in phase space caused by surface hops. We stress that for each trajectory the electronic density matrix is fully coherent, i.e., it is only when the entire ensemble is averaged that the two sources of decoherence mentioned above arise.

The second general class of methods includes self-consistent potential (SCP) methods, ${ }^{1}$ in which the nuclei evolve on an effective time-dependent potential energy surface that depends on the current quantum mechanical electronic density matrix. The simplest version of this approach is the semiclassical Ehrenfest (SE) method, ${ }^{6-9}$ and the most highly developed versions are the mean field with surface hopping $(\mathrm{MF} / \mathrm{SH})$ method $^{10}$ and the selfconsistent decay of mixing (SCDM) algorithm. ${ }^{11}$ The SE method involves an ensemble of trajectories and therefore includes some decoherence due to the initial spread of the wave packet. The electronic density matrix for each trajectory in the ensemble is propagated with full coherence, as discussed above for the TSH method. Furthermore, SE trajectories evolve under the influence of all of the electronic states at once according to the electronic state density matrix (i.e., there is no hopping between states) such that each trajectory in the ensemble is fully coherent. During a rapid passage of a single region of strong coupling, one may expect fully coherent nuclear motion, and the SE approach is reasonable for that case. The lack of decoherence, however, leads to nonphysical behavior in the asymptotic regions of the simulations (i.e., as the system comes into the interaction region from reactants or goes 
toward products) where the coupling is often small or zero; this contrasts with the TSH approach, which leads to physical asymptotic behavior but may be less accurate in describing strongly coupled motions.

The SCDM method introduces the physical asymptotic behavior of a TSH method into an SCP method. In particular, decoherence (discussed above only as an effect of ensemble averaging) is introduced explicitly into the equations of motion for each individual trajectory in an average way, while still propagating each trajectory independently. This modification results in an SE-like trajectory with the correct limiting behavior.

Alternatively one might try to improve upon TSH methods by making them more coherent. This leads to a class of methods that may be called coherent passages methods. In coherent passage methods, a trial classical path with continuous momenta is used to propagate the system through an entire strong coupling region with a fully coherent treatment of the electronic density matrix (i.e., without surface hopping and without decay of mixing). Then, the electronic transition probabilities from this coherent evolution are used in some fashion to control the trajectory outcome, but subsequent passages through this or any other strong interaction region are not treated as coherent with the previous passage. The importance of decoherence between successive passages through a strong interaction region has been demonstrated most clearly by Thachuk et al. ${ }^{12}$ in a low-dimensionality problem, namely the evolution of a two-state diatomic molecule in a strong electromagnetic field; their discussion is very enlightening and makes it clear that the combination of coherent evolution through a strong interaction region and decoherence between such passages can also be important in the general case (although their algorithm is not general). Their examples make it especially clear that maintaining coherence over an entire trajectory can lead to significant 
errors. One classical path method that was developed with this kind of consideration as a motivation is the surface hopping method of Parlant and Gislason, ${ }^{13}, 14$ which differs from an earlier method of Kuntz et al. 15 in the prescription for calculating the hopping probability and from an earlier method of Blais and Truhlar ${ }^{16}$ in its insistence on coherent evolution through each "complete passage" la of a strong coupling region. This method, therefore, mitigates some of the decoherence that arises (in other TSH methods) from surface hops within strongly coupled regions as well as the inaccuracies explained by Thachuk et al. ${ }^{12}$ that arise from treating successive passages coherently. Parlant and Gislason ${ }^{13}$ called their method the "exact" surface hopping scheme because it uses the exact time-dependent electronic Schrödinger equation for electronic motion in each complete passage through a region of strong coupling. This could be confusing because a variety of surface hopping methods and other semiclassical methods use the exact time-dependent electronic Schrödinger equation in one or another way as part of the algorithm; to emphasize the special character of the Parlant-Gislason method we call it "exact complete passage" trajectory surface hopping (ECP-TSH).

The methods discussed so far are independent trajectory methods. Another class of methods for non-Born-Oppenheimer trajectories involves coupled trajectories, where an entire swarm of trajectories is evolved simultaneously in such a way that the entire electronic state density matrix influences the motion of each trajectory. This is a very reasonable approach because it is the ensemble of trajectories, not the individual phase points, that should be interpreted when one semiclassically infers quantum mechanical transition probabilities from classical mechanics. The most thoroughly developed coupled-trajectory schemes are the quantum/classical Liouville methods of Martens and coworkers ${ }^{17}$ and 
others ${ }^{18}$ and the full multiple spawning method. ${ }^{19}$ Like SCDM, this class of methods explicitly includes dynamical decoherence, but it is more complicated than the independenttrajectory methods, and coupled-trajectory methods require considerably more computational effort to fully sample the extended phase space of both the nuclear coordinates and momenta and the electronic probability amplitudes. Therefore, although they are very promising, we will not consider coupled-trajectory methods further in the present article.

Our recent work has involved refining both the TSH and SCP approaches, leading respectively to the recent developments of the $\mathrm{FSTU}^{4}$ and $\mathrm{SCDM}^{11}$ methods. We emphasize that, in the context of TSH, the TFS and FSTU surface hopping schemes both allow hops even in the middle of a single passage of a strong coupling region, and, in the context of SCP calculations, the SCDM scheme allows some decay of mixing at all points along a trajectory, even for the equations determining the probability of switching the decoherent state, and therefore these methods do not conform to the coherent passage ansatz. It is not clear from previous work how important this might be for multi-dimensional problems since Refs. 12 and 13 only tested the importance of coherent complete passage for low-dimensional problems where phases, coherence, and quantum mechanical interference effects are not subject to even the minimal averaging inherent in collisions of molecules when one does not initially select and finally analyze the angular momentum projection quantum numbers. Therefore in this article we consider coherent complete passage methods in more detail and compare their performance to that of the TFS, FSTU, SE, and SCDM methods. In particular we examine two such methods, the ECP-TSH method ${ }^{11}$ discussed above and a new coherentpassage-type method obtained by employing a coherent switching algorithm in SCDM for each complete passage of a strong coupling region. We call the new method coherent 
switches with decay of mixing or CSDM. We also test a variant, explained below, called CSDM-C.

Section II reviews fundamental time-dependent coupled equations for the motion of the electrons and nuclei that are used by all of the methods tested in this paper. Section III reviews the ECP-TSH method and compares its equations to those for the TFS and FSTU methods. Section IV presents the new CSDM method. Section V presents the methodology and comparison of several semiclassical trajectory methods for five three-dimensional atommolecule systems. Section VI presents the results and discussion. Section VII summarizes the main conclusions.

\section{FUNDAMENTAL TIME-DEPENDENT EQUATIONS}

All of the methods tested in this paper employ the time-dependent electronic Schrödinger equation, which may be written as follows in terms of the elements $\rho_{k k^{\prime}}$ of the electronic density matrix: ${ }^{11}$

$$
i \hbar \dot{\rho}_{k k^{\prime}}=\sum_{l}\left(\rho_{l k^{\prime}}\left[U_{k l}-i \hbar \dot{\mathbf{R}} \cdot \mathbf{d}_{k l}\right]-\rho_{k l}\left[U_{l k^{\prime}}-i \hbar \dot{\mathbf{R}} \cdot \mathbf{d}_{l k^{\prime}}\right]\right),
$$

where $k$ and $k^{\prime}$ label electronic states $\left(k, k^{\prime}=1,2, \ldots, m\right.$, where $m$ is the number of electronic states), $\mathbf{R}$ is an $\mathrm{N}$-dimensional vector of nuclear coordinates, an overdot denotes a time derivative, and $U_{k k^{\prime}}$ are the matrix elements of the electronic Hamiltonian $H_{\mathrm{el}}$ (which includes nuclear-nuclear repulsion):

$$
U_{k k^{\prime}}=\left\langle k\left|H_{\mathrm{el}}\right| k^{\prime}\right\rangle .
$$

The diagonal elements of $U_{k k^{\prime}}$ are called potential energy surfaces, and the off-diagonal elements couple motion in the various electronic states. The eigenvalues of $\mathbf{U}$ are the 
adiabatic potential energy surfaces, called $V_{k}$. The nonadiabatic coupling vector $\mathbf{d}_{k k^{\prime}}$ is an $m$ $\times m$ anti-Hermitian matrix in electronic state space, and each element is a vector in $\mathbf{R}$ :

$$
\mathbf{d}_{k k^{\prime}}=\left\langle k\left|\nabla_{\mathbf{R}}\right| k^{\prime}\right\rangle
$$

where $\nabla_{\mathbf{R}}$ is the $N$-dimensional nuclear gradient. We solve the equations in an isoinertial, mass-scaled nuclear coordinate system $\mathbf{R}$ in which all nuclear masses are scaled to the same reduced mass $\mu$. The momentum conjugate to $\mathbf{R}$ is called $\mathbf{P}$. In the adiabatic representation, $\mathbf{U}$ is a diagonal matrix called $\mathbf{V}$; and one can define a "diabatic" representation where $\mathbf{d}_{k k^{\prime}}$ is zero and $\mathbf{U}$ is not diagonal. Strictly diabatic representations do not exist, ${ }^{20}$ but representations in which $\mathbf{d}_{k k^{\prime}}$ is small enough to be neglected are very useful and, following a widespread practice in the field, will be called diabatic in the present paper.

The semiclassical Hamiltonian that governs nuclear motion can be written

$$
H_{\mathrm{C}}=\frac{\mathbf{P}^{2}}{2 \mu}+V^{\mathrm{E}}
$$

where $V^{\mathrm{E}}$ is an effective potential energy surface, and its form depends on the specific semiclassical trajectory method and how that method treats non-Born-Oppenheimer effects. The nuclear motion is represented by a swarm of classical trajectories, and the nuclear position and momentum of each trajectory evolves according to classical equations of motion,

$$
\begin{gathered}
\dot{\mathbf{R}}=\mathbf{P} / \mu \\
\dot{\mathbf{P}}=-\nabla_{\mathbf{R}} V^{\mathrm{E}} .
\end{gathered}
$$

We note that the electronic Schrödinger equation may be formulated in the particle representation where electronic action and angle variables are transformed to particle-like 
generalized coordinates and momenta, ${ }^{6}$ or in the density representation of Eq. (1). Although the two representations are equivalent in the case of two electronic states, the particle representation requires an adiabatic-diabatic transformation that can be ambiguous for the case of more than two electronic states. ${ }^{21}$ Throughout the present paper, we use the density representation as in Eq. (1).

We assume that the adiabatic and diabatic representations are the same in asymptotic regions, so that both $U_{k k^{\prime}}$ with $k \neq k^{\prime}$ and $\mathbf{d}_{k k^{\prime}}$ vanish asymptotically.

In TSH methods, $V^{E}$ is given by a single potential energy surface (i.e., an adiabatic potential energy surface $V_{k}$ or a diabatic potential energy surface $U_{k k}$ in the adiabatic and diabatic representations, respectively), and $k$ may switch to some other state $k^{\prime}$ at certain points along the trajectory according to some hopping probability $g_{k k^{\prime}}$, which is a function of the electronic state density matrix $\rho$ and its time derivative. Except possibly at hops (or frustrated hops explained below), $\boldsymbol{\rho}$ is obtained by integrating Eq. (1) without any

modifications. In SCP methods, $V^{E}$ is more complicated, and in SCP methods that include decay of mixing, one also modifies Eq. (1). We will consider SCP methods further in Section IV.

\section{ECP-TSH METHOD}

In trajectory surface hopping (TSH) methods, nonadiabatic transitions are treated as discontinuous hops (or switches) from one potential surface to another. The electronic coupled equations (1) are integrated along a classical trajectory and are used to determine the location of hops. In general, trajectory surface hopping methods involve trajectories that hop 
back and forth between potential energy surfaces, and they differ in their prescription for how to do so. In Sec. I, we mentioned that the most satisfactory versions of the well-tested TSH approaches are the TFS method ${ }^{3}$ and the FSTU method, ${ }^{4,5}$ which is an extension of the TFS method that improves the treatment of frustrated hops, which are tentative hops forbidden by energy or momentum conservation. Both TFS and FSTU, in principle, allow a trajectory to hop whenever $\rho_{k k}$ is changing. On the other hand, the ECP-TSH method, developed by Parlant and Gislason, ${ }^{13}$ is defined in such that a trajectory is allowed to hop only where the coupling is locally maximum. For instance, in the two-state case the ECPTSH method uses

$$
\Omega(t)=\left|\dot{\mathbf{R}} \cdot \mathbf{d}_{12}(t)\right|
$$

as a measure of the strength of the coupling. Hops are allowed at positions along a trajectory where there is a local maximum of this coupling strength function. Furthermore, the electronic density is reinitialized at all local minima of $\Omega$, i.e., $\rho_{k k}$ is set to unity where $k$ is the currently occupied electronic state, and all other $\rho_{k^{\prime} k^{\prime \prime}}$ are set to zero. The hopping probability from state $k$ to $k^{\prime}$ at each maximum of $\Omega$ is determined by integrating along a trajectory between the two adjacent local minima of $\Omega$, i.e., by integrating from one local minimum at $t_{i-1}$ to another at $t_{i}$, where $t_{i}$ is $i$ th local minimum of $\Omega(t)$. In particular, before it is reinitialized, $\rho_{k k}\left(t_{i}\right)$ determines the hopping probability at the local maximum of $\Omega$ between $t_{i-1}$ and $t_{i}$. In the ECP-TSH method, a trajectory undergoes complete coherent passage between two adjacent local minima of $\Omega$ before the trajectory is brought back to the maximum of $\Omega$ to allow for a hop. After the attempted hop, the coherence $\rho_{k k^{\prime}}$ is destroyed 
as $\rho_{k k^{\prime}}$ is reinitialized. This is different from TFS and FSTU where $\rho_{k k^{\prime}}$ is never reinitialized and is therefore fully coherent along each entire trajectory.

Note that $\Omega$ tends to zero as $t \rightarrow \infty$ after the collision (or photodissociation event). This is interpreted as a local minimum, and so the trajectory can hop at the final local maximum of $\Omega$. Similarly $t_{0}$ is defined as the start of the trajectory so that a hop can occur at the first local maximum for $\Omega$.

The original ECP-TSH applications by Parlant and Gislason ${ }^{13}$ used the so-called "ants" sampling scheme, where at each hopping location the trajectory is split into two weighted branches. Both branches are propagated independently on the two potential energy surfaces, and each branch may undergo additional future branching. Later, Parlant and Alexander ${ }^{14 \mathrm{a}}$ applied a mixed anteater/ants scheme, and Sizun et al. ${ }^{14 \mathrm{~b}}$ used the anteater sampling scheme where only one branch is followed. The sampling algorithm involves a cutoff parameter $\Omega_{\text {cutoff }}$; only maxima with $\Omega$ greater than $\Omega_{\text {cutoff }}$ are recognized as possible hopping locations. We apply the anteater implementation of the ECP-TSH method, recognizing that for a large enough ensemble of trajectories both the ants and anteater implementations should give the same results.

Although the original ECP-TSH method ${ }^{13}$ was formulated in the adiabatic representation, there is no reason why it cannot be applied in the diabatic representation. Note that $\mathbf{d}_{12}$ is given as a function of the matrix element of $\mathbf{U}$ by Eq. (A9) of Ref. 22, and in the two-state case, the nonadiabatic coupling vector $\mathbf{d}_{12}$ provides an equally good measure of the coupling in the diabatic representation as in the adiabatic one. ${ }^{11}$ In the present paper we therefore extend the ECP-TSH method to the diabatic representation by using the same 
scheme as is in the adiabatic representation based on the same reference equation (7). This extension will be tested for the test cases in Sec. V.

\section{SELF-CONSISTENT POTENTIAL METHODS}

In the self-consistent decay of mixing (SCDM) method, the effective potential energy surface $V^{\mathrm{E}}$ is

$$
V^{\mathrm{E}}=\sum_{k} \rho_{k k} U_{k k}+\sum_{k} \sum_{k^{\prime}<k} 2 \operatorname{Re}\left(\rho_{k k^{\prime}}\right) U_{k k^{\prime}}
$$

Recall that in the adiabatic representation $U_{k k}=V_{k}$ and $U_{k k^{\prime}}=0$ if $k \neq k^{\prime}$, whereas in the diabatic representation the second term of Eq. (8) is nonzero. In the SCDM method, the electronic density evolves by

$$
\dot{\rho}_{i j}=\dot{\rho}_{i j}^{C}+\dot{\rho}_{i j}^{D}
$$

in which the first part comes from fully coherent contribution in Eq. (1), and the second part is the decay of mixing term: 11

$$
\dot{\rho}_{i i}^{D}= \begin{cases}-\frac{\rho_{i i}}{\tau_{i K}}, & i \neq K \\ \sum_{j \neq K} \frac{\rho_{j j}}{\tau_{K j}}, & i=K\end{cases}
$$

for the diagonal elements, and

$$
\dot{\rho}_{i j}^{D}=\left\{\begin{array}{cc}
-\frac{1}{2}\left(\frac{1}{\tau_{i K}}+\frac{1}{\tau_{j K}}\right) \rho_{i j}, & i \neq K, \quad j \neq K \\
\frac{1}{2}\left(\frac{1}{\rho_{K K}} \sum_{k \neq K} \frac{\rho_{k k}}{\tau_{K k}}-\frac{1}{\tau_{j K}}\right) \rho_{i j}, & i=K, \quad j \neq K \\
\frac{1}{2}\left(\frac{1}{\rho_{K K}} \sum_{k \neq K} \frac{\rho_{k k}}{\tau_{K k}}-\frac{1}{\tau_{i K}}\right) \rho_{i j}, & i \neq K, \quad j=K
\end{array}\right.
$$


for the off-diagonal elements. Detailed derivations of Eqs. (10) and (11) are given in Ref. 11. The equations of motion for the nuclear position are given by Eq. (5), and those for the momentum are

$$
\dot{\mathbf{P}}=\dot{\mathbf{P}}^{\mathrm{C}}+\dot{\mathbf{P}}^{\mathrm{D}}
$$

where the first term is the instantaneous change in momentum due to the fully coherent nonadiabatic motion determined by Eqs. (1), (6), and (18):

$$
\begin{aligned}
\dot{\mathbf{P}}^{\mathrm{C}}(t) & =-\sum_{k} \rho_{k k} \nabla_{\mathbf{R}} U_{k k}-\sum_{k} \sum_{k^{\prime}<k}\left(2 \operatorname{Re} \rho_{k k^{\prime}}\right) \nabla_{\mathbf{R}} U_{k k^{\prime}} \\
& +\sum_{j} \sum_{k} \sum_{k^{\prime}}\left(2 \operatorname{Re} \rho_{k j}\right) U_{k k^{\prime}} \mathbf{d}_{k^{\prime} j}
\end{aligned}
$$

In the diabatic representation, Eq. (13) becomes

$$
\dot{\mathbf{P}}^{\mathrm{C}}(t)=-\sum_{k} \rho_{k k} \nabla_{\mathbf{R}} U_{k k}-\sum_{k} \sum_{k^{\prime}<k}\left(2 \operatorname{Re} \rho_{k k^{\prime}}\right) \nabla_{\mathbf{R}} U_{k k^{\prime}}
$$

and in the adiabatic representation, Eq. (13) becomes

$$
\dot{\mathbf{P}}^{\mathrm{C}}(t)=-\sum_{k} \rho_{k k} \nabla_{\mathbf{R}} V_{k}+\sum_{k} \sum_{k^{\prime}} \operatorname{Re}\left(\rho_{k k^{\prime}}\right)\left(V_{k}-V_{k^{\prime}}\right) \mathbf{d}_{k k^{\prime}}
$$

The second term in Eq. (12) is the decoherent force and is given by

$$
\dot{\mathbf{P}}^{\mathrm{D}}=-\frac{\mu \dot{V}^{\mathrm{D}}}{\mathbf{P} \cdot \hat{\mathbf{s}}} \hat{\mathbf{s}}
$$

with $^{23}$

$$
\dot{V}^{\mathrm{D}}=\sum_{k} \dot{\rho}_{k k}^{D} U_{k k}+\sum_{k} \sum_{k^{\prime}<k} 2 \operatorname{Re}\left(\dot{\rho}_{k k^{\prime}}^{D}\right) U_{k k^{\prime}}
$$

The force in Eq. (16) drives the trajectory to a pure electronic state. The unit vector $\hat{\mathbf{s}}$ represents the direction into which energy is deposited and out of which energy is consumed, and it is considered in subsection IV. D. The decoherent state switches during the trajectory 
with the switches governed by a switching probability that will be discussed in Subsections IV.A to IV.C.

\section{IV.A. NDM}

The natural decay of mixing (NDM) method 23 computes the switching probability by the most naïve application of the fewest switches ${ }^{3}$ criterion. For example, in the two-state case, the probability of switching from decoherent state $K$ to some other state $K^{\prime}$ between time $t$ and time $t+d t$ is given by

$$
P_{K \rightarrow K^{\prime}}=\max \left(-\frac{\dot{\rho}_{K K} d t}{\rho_{K K}}, 0\right)=\max \left(-\frac{\left(\dot{\rho}_{K K}^{C}+\dot{\rho}_{K K}^{D}\right) d t}{\rho_{K K}}, 0\right)
$$

The multi-state generalization of Eq. (18) is given in Eq. (A1) of Appendix A.

\section{IV.B. SCDM}

As pointed out previously, ${ }^{11} \dot{\rho}_{K K}^{D}$ in Eq. (18) is always positive, and this causes a system modeled using the NDM method to artificially resist changing its decoherent state. The SCDM method makes a simple modification to Eq. (18) by dropping the decoherent part in the numerator, i.e., the switching probability is given by

$$
P_{K \rightarrow K^{\prime}}=\max \left(-\frac{\dot{\rho}_{K K}^{C} d t}{\rho_{K K}}, 0\right)
$$

This is called the self-consistent switching probability and may be interpreted as "locally coherent”. Appendix A contains the multi-state generalization of Eq. (19). 


\section{IV.C. CSDM}

The electronic density matrix elements $\rho_{k k^{\prime}}$ in the decay of mixing algorithm vary along the trajectory and depend on the location of decoherent state switches. If one of these switches occurs during a traversal of a strong interaction region, the state change influences the dynamics of $\rho_{k k^{\prime}}$ in the remainder of that region. If we define coherence as the evolution of the density matrix according to Eq. (1) with no external influence on the trajectory, then the SE method is coherent, but either decay of mixing or switches in the decoherent state destroy coherence. The effective decoherence in the SCDM method is less severe than that in the NDM method; since this has been found to improve the results, ${ }^{11}$ it is interesting to consider a method that fully removes the decoherence from the electronic state equations for each strong interaction region; this is called the CSDM method. In the CSDM method, the switching probability is controlled by a fully coherent solution of Eq. (1) in each complete passage of a strong interaction region. However, the effective potential for nuclear motions is treated as in the SCDM method.

Following Parlant and Gislason ${ }^{13}$ and Thachuk et al., ${ }^{12}$ we modify the switching scheme to treat each complete passage of a strong coupling region coherently. We define

$$
D_{K}(t)=\sum_{j}\left|\mathbf{d}_{K j}\right|^{2}
$$

and

$$
C_{K}(t)=\sum_{j}\left|\mathbf{d}_{K j} \cdot \dot{\mathbf{R}}_{\mathrm{vib}}\right|^{2} .
$$


We also define a coherent set of state populations $\widetilde{\rho}_{K K^{\prime}}$. As opposed to $\rho_{K K^{\prime}}$, which evolves by the decay of mixing Eqs. (9)-(11) and which is used in Eqs. (8) and (13)-(17), $\widetilde{\rho}_{K K^{\prime}}$ evolves by the completely coherent equation (1). At each local minimum of $D_{K}(t)$ along trajectory, we set $\widetilde{\rho}_{i j}=\rho_{i j}$ for all $i$ and $j$, and the probability of switching the decoherent state is given by

$$
P_{K \rightarrow K^{\prime}}=\max \left(-\frac{\dot{\tilde{\rho}}_{K K} d t}{\widetilde{\rho}_{K K}}, 0\right)
$$

This is called coherent switches with decay of mixing or CSDM. In the multistate case, Eq. (A1) replaces Eq. (22). Alternatively, we could set $\widetilde{\rho}_{i j}=\rho_{i j}$ at each local minimum of $C_{K}$, and this is called CSDM based on a component of nonadiabatic coupling or CSDM-C.

We emphasize that the equations of motion governing the $\widetilde{\rho}_{i j}$ elements and hence governing the switching probability in the CSDM method are treated in a coherent and uninterrupted way throughout each complete passage through a strong coupling region (although one does allow switches in the decoherent state), but decoherence is introduced into $\widetilde{\rho}_{i j}$ between different strong coupling regions by setting $\widetilde{\rho}_{i j}=\rho_{i j}$; the ECP-TSH method also integrates the electronic equations of motion in a coherent way throughout each complete transversal of a strong coupling region, but it handles decoherence differently in several respects. First of all, there are no decay of mixing terms. Second, the ECP-TSH method involves hops with discontinuities in the nuclear momentum, and when a hop occurs it requires that one goes back to a point of maximal coupling, whereas the CDSM algorithm never goes back to an earlier point in the trajectory. Third, and this may be very important, in 
the ECP-TSH algorithm, whether or not a hop occurs, at each local minimum of $\Omega(t)$, the electronic coefficients are reinitialized to unity or zero. The analog in CSDM would be to set $\widetilde{\rho}_{i j}=\delta_{i j} \delta_{i K}$ rather than $\widetilde{\rho}_{i j}=\rho_{i j}$. By setting $\widetilde{\rho}_{i j}$ equal to $\rho_{i j}$, the amount of decoherence is determined by the difference between the two electronic density matrices: $\boldsymbol{\rho}$, which is propagated with decay-of-mixing terms, and $\tilde{\boldsymbol{\rho}}$, which is propagated coherently. Thus, in particular, the amount of decoherence introduced by CSDM at a local minimum of $D_{K}(t)$ depends on the size of the coupling region and other dynamical factors, whereas ECPTSH fully destroys the coherence between any two strong coupling regions, no matter what their character is.

The NDM, SCDM, CSDM, and CSDM-C methods differ from each other only in the scheme that switches the decoherent state. The NDM method uses the decay-of-mixing electronic density to calculate the switching probability; the SCDM method eliminates the decoherent part of the electronic density locally in the switching algorithm; and the CSDM and CSDM-C methods, in contrast, switch off the contribution of the decoherence to the electronic density matrix used in the switching probability over an entire region of strong coupling.

It is also interesting to comment on the relationship of the CSDM method and the ECP-TSH method to the method of Kuntz, ${ }^{24}$ which he has called the classical path surface hopping (CPSH) method. Unlike ECP-TSH, which combines complete coherent passage with surface hopping, or CSDM, which combines them with a self-consistent potential scheme, CPSH attempts combine all three approaches. In particular Kuntz uses the semiclassical Ehrenfest method in a given strong interaction region, followed by decay of mixing between strong interaction regions, after the last strong interaction region, or when the probability in 
closed states is too high. (Although Kuntz calls this decay of mixing "surface hopping', it is achieved in practice by adding decay terms to the Ehrenfest equation. The decay time is set equal to 0.25 times a monitoring interval (see Eq. (14) in Ref. 14a). The monitoring interval is set equal to "one program unit," $24 \mathrm{~b}$ which equals $5.4 \mathrm{fs}$, or to "a short time interval." $24 \mathrm{~d}$ Because of the way that the decay terms are employed, it is not unreasonable to think of the decay segments as smoothed surface hops between or following Ehrenfest regions.) For a system with a single strong interaction region the CPSH method becomes similar to the semiclassical Ehrenfest method with linear smooth sampling 9 (SE-LSS). Since we have previously shown that SCDM and NDM are more accurate than SE-LSS 11,23 and that SCDM is more accurate than NDM, ${ }^{11}$ we have only tested adding the coherent-complete-passage refinement to SCDM, not to the SE or NDM methods. Furthermore we note that Kuntz compared the CPSH method to ECP-TSH and concluded ${ }^{14 a}$ that the two methods ought to produce very similar results since the methods both integrate Eq. (1) through the strong interaction region and differ only in technical aspects. Since we carry out a complete test of the ECP-TSH method, which has the advantage of not involving an undetermined monitoring interval, we do not test the later, more complicated CPSH method.

\section{IV.D. Decoherent direction}

In SCDM, the decoherent direction was originally given by ${ }^{11}$

$$
\hat{\mathbf{s}}=\left(d_{K k} a_{0} P_{K k}^{(\mathbf{d})} \hat{\mathbf{d}}_{K k} \pm P_{\mathrm{vib}} \hat{\mathbf{P}}_{\mathrm{vib}}\right) /\left\|d_{K k} a_{0} P_{K k}^{(\mathbf{d})} \hat{\mathbf{d}}_{K k} \pm P_{\mathrm{vib}} \hat{\mathbf{P}}_{\mathrm{vib}}\right\|,
$$

where $a_{0}$ is a bohr length, $\hat{\mathbf{P}}_{\mathrm{vib}}$ and $\hat{\mathbf{d}}_{K k}$ are unitless unit vectors in the direction of $\mathbf{P}_{\mathrm{vib}}$ (the local vibrational momentum ${ }^{22}$ ) and $\mathbf{d}_{K k}$, respectively, $d_{K k}$ is the magnitude of $\mathbf{d}_{K k}, P_{K k}^{(\mathbf{d})}$ 
is the component of $\hat{\mathbf{P}}_{\mathrm{vib}}$ in the direction of $\mathbf{d}_{K k}$, and $K$ is the decoherent state. The sign in Eq. (23) was chosen such that the summation is additive. Since both $\mathbf{d}_{K k}$ and $\mathbf{P}_{\text {vib }}$ are within the non-rotational subspace, using $\hat{\mathbf{s}}$ as the decoherent direction conserves total angular momentum. There is some ambiguity is separating vibrational and rotational motion, i.e., in the definition of $\mathbf{P}_{\mathrm{vib}}$. To eliminate this ambiguity, we replace $P_{K k}^{(\mathbf{d})}$ with $\mathbf{P} \cdot \hat{\mathbf{d}}_{K k}$ (i.e., the component of total momentum in the direction of $\mathbf{d}_{K k}$ ). This yields

$$
\hat{\mathbf{s}}=\left(d_{K k} a_{0}\left(\mathbf{P} \cdot \hat{\mathbf{d}}_{K k}\right) \hat{\mathbf{d}}_{K k} \pm P_{\mathrm{vib}} \hat{\mathbf{P}}_{\mathrm{vib}}\right) /\left\|d_{K k} a_{0}\left(\mathbf{P} \cdot \hat{\mathbf{d}}_{K k}\right) \hat{\mathbf{d}}_{K k} \pm P_{\mathrm{vib}} \hat{\mathbf{P}}_{\mathrm{vib}}\right\| .
$$

Equations (23) and (24) are for the two-state case; see Ref. 11 for a generalization of Eq. (23) to multiple states in both adiabatic and diabatic representations, and we can generalize Eq. (24) in the same way.

\section{IV.E. Decay-of-mixing time}

In general, the SCDM and CSDM algorithms presented above may be used with a variety of models for the decay-of-mixing times $\tau_{i K}$. For example, we previously ${ }^{11,23}$ presented simple models based primarily on phase decay and on a combination of phase decay and the requirement that demixing should vanish in the limit of low nuclear momentum. In addition, Fiete and Heller and Turi and Rossky have presented treatments based on short-time ${ }^{25}$ or perturbative 26 treatments of Gaussian wave packets. These treatments provide general guides to the form of the physical decoherence function; however, we need to emphasize that physical decoherence and algorithmic demixing are closely related but quantitatively different. Algorithmic demixing is the decay of the reduced density matrix elements (off-diagonal elements to zero and diagonal elements to 
zero or unity) that must be added to a quantum/classical algorithm in order that a calculation based on an ensemble of independent trajectories of the classical subsystem simulates, as well as possible, the evolution of a real system in which both electronic and nuclear coordinates are quantum mechanical. A single SCP trajectory has a physical nuclear kinetic energy only if the electronic state is pure. But a quantum mechanical reduced density matrix generally corresponds to a mixture even when the whole system is in a pure state; it corresponds to a pure state only if the whole system is in an unentangled pure state. Thus, in order to obtain a practical semiclassical algorithm based on independent trajectories, we must devise an algorithmic decay of mixing that does not correspond precisely to quantum mechanical decoherence.

Although future work may develop more sophisticated semiclassical justifications of the appropriate decay of mixing rates that should be used, the calculations presented here are based on the assumption that we can use the simplest treatment that satisfies the following two constraints: (1) At low values of $\mathbf{P} \cdot \hat{\mathbf{s}}$, Eq. (16) requires that

$$
\frac{1}{\tau_{i K}} \stackrel{\mathbf{P} \cdot \hat{\mathbf{s}} \rightarrow 0}{\sim}(\mathbf{P} \cdot \hat{\mathbf{s}})^{n}, n>1
$$

in order that demixing does not occur when the momentum in the direction that couples electronic and nuclear motion is insufficient to support the required accompanying energy transfer, and (2) the demixing time should not be shorter than the shortest electronic time scale in the problem, which we take to imply that

$$
\frac{1}{\tau_{i K}} \leq \frac{\left|V_{i i}-V_{K K}\right|}{\hbar} .
$$

Two of the simplest possible functions that satisfy these constraints are 


$$
\tau_{i K}=\hbar\left(\frac{C}{\left|V_{i i}-V_{K K}\right|}+\frac{C^{\prime}}{(\mathbf{P} \cdot \hat{\mathbf{s}})^{2} / 2 \mu}\right)
$$

and

$$
\tau_{i K}=\frac{\hbar}{\left|V_{i i}-V_{K K}\right|}\left(C+\frac{E_{0}}{(\mathbf{P} \cdot \hat{\mathbf{s}})^{2} / 2 \mu}\right)
$$

where $C$ and $C^{\prime}$ are unitless parameters greater than or equal to unity, and $E_{0}$ is a positive parameter with units of energy. Equation (27) is similar to the form we used in our previous paper, ${ }^{11}$ and equation (28) with $C=0$ is similar to the form we used in our original $^{23}$ decay-of-mixing paper. In the present work, we have tested various forms of this type, with $\mathrm{C}=1$ through 5 , and we have found that the results are not very sensitive to the particular form of $\tau_{i K}$ or to the values of the parameters, provided only that $\tau_{i K}$ is large enough. As an example, in Appendix B we present full sets of results with three values of $E_{0}$ which illustrate the insensitivity of the results to this parameter. The results are even less sensitive to $C$. Therefore, we simply use Eq. (28) with $C=1$ and $E_{0}$ equal to 0.1 hartree.

All of the decay-of-mixing results given in this paper follow the formalism of Eqs. (8)-(22), (24), and (28) and differ only in how the switching probability is calculated.

\section{THREE-DIMENSIONAL TEST CASES AND METHODOLOGY}

We apply the SCDM, CSDM, CSDM-C, and ECP-TSH methods to five fullydimensional model systems with various initial conditions for a total of twelve test cases, as discussed in Ref. 11. Descriptions of the model surfaces and details of the accurate quantum mechanical calculations have been previously presented for the $\mathrm{MXH}^{27}$ and $\mathrm{YRH}^{28}$ systems. 
Briefly, each model system has two electronic states and is defined in terms of a diabatic potential energy matrix (PEM), which includes two diagonal potential energy surfaces and a coupling surface. The electronically nonadiabatic atom-diatom collisions that comprise our test suite all have the form:

$$
\mathrm{A} *+\mathrm{BC}(v, j) \rightarrow\left\{\begin{array}{l}
\mathrm{B}+\mathrm{AC}\left(E_{\mathrm{int}}^{\prime}\right) \\
\mathrm{A}+\mathrm{BC}\left(E_{\mathrm{int}}^{\prime \prime}\right)
\end{array}\right.
$$

where $(A, B, C)=(M, H, X)$ and $(Y, R, H)$ for the MXH and YRH systems, respectively, the asterisk indicates electronic excitation, $v$ and $j$ are the initial vibrational and rotational quantum numbers, and the final internal (i.e., rovibrational) energy of the diatomic fragment is $E_{\text {int }}^{\prime}$ for reaction products and $E_{\text {int }}^{\prime \prime}$ in the quenched arrangement.

We label the initial conditions by the total energy $E$ given in $\mathrm{eV}$ and the initial rotational state $j$ of the diatomic molecule $[i . e .$, by $(E / \mathrm{eV}, j)]$. For all of the cases considered here, the diatom is initially in its ground vibrational state (i.e., $v=0$ ), and the total angular momentum of the system is zero. Electronic angular momentum is neglected.

The nine MXH cases are: the SB, SL, and WL parameterizations of the MXH system with the masses of the $\mathrm{M}, \mathrm{H}$, and $\mathrm{X}$ model atoms equal to $6.04695,1.00783$, and 2.01565 amu, respectively, and with $(1.10,0),(1.10,1)$, and $(1.10,2)$ initial conditions. Details of the MXH parameterizations and initial conditions are given in Ref. 27.

The three YRH cases are: the $\mathrm{YRH}(0.1)$ parameterization with the $(1.10,0)$ initial conditions, and the $\mathrm{YRH}(0.2)$ parameterization with the $(1.02,0)$ and $(1.10,6)$ initial conditions. The masses of the $\mathrm{Y}, \mathrm{R}$, and $\mathrm{H}$ atoms are 10, 6, and $1.00783 \mathrm{amu}$, respectively. Details of the YRH parameterizations and initial conditions are given in Ref. 28. 
Since we do not expect the semiclassical methods to reproduce quantum mechanical oscillations in most cases, and since these oscillations usually wash out in experimental observables, our goal is to learn how well the semiclassical results can reproduce the quantal ones when any oscillations in the quantal results are averaged out. Therefore, the results from quantum mechanical calculations at several energies at and around the nominal scattering energy were calculated and averaged (full details are given elsewhere $11,27,28$ ). In almost all cases, the values obtained by averaging are very similar to the values obtained at the nominal energy.

For the methods involving trajectories, the coordinates and momenta of the nuclei and the electronic state populations were integrated using an adaptive integration algorithm that was designed for use with semiclassical trajectory calculations. ${ }^{22}$ The algorithm uses a Bulirsch-Stoer integrator with polynomial extrapolation 29,30 modified such that the integrator is prohibited from stepping over local peaks and minima in the electronic probabilities. For the present calculations, the integration parameters ${ }^{27}$ were given the following values: $\varepsilon_{\mathrm{BS}}=$ $10^{-12} E_{\mathrm{h}}\left(1 E_{\mathrm{h}}=27.211 \mathrm{eV}\right)$ and $h_{\min }=10^{-4}$ a.u. $\left(1\right.$ a.u. $\left.=2.4189 \times 10^{-2} \mathrm{fs}\right)$, which give converged results for the YRH and MXH systems. The trajectories begin the simulation with the lone atom ( $\mathrm{Y}$ in the case of YRH and $\mathrm{M}$ in the case of MXH) separated from the centerof-mass of the diatom by $35 \mathrm{a}_{0}\left(1 \mathrm{a}_{0}=0.52918 \AA\right)$ for the MXH cases and by $20 \mathrm{a}_{0}$ for the YRH cases, and the simulation was ended when the product fragments were separated by at least $30 \mathrm{a}_{0}$ for both systems. We have verified that the results of the semiclassical simulations do not change when these distances are increased.

For the methods involving trajectories, the final state internal energies $E_{\mathrm{int}}^{\prime}$ or $E_{\mathrm{int}}^{\prime \prime}$, were determined without quantization. In particular, in all trajectory methods, the relative 
translational energy and the electronic energy become constant after the collision, and the internal energy is computed as total energy minus final relative translational energy minus final electronic energy. (Note that, for the problems considered here, $U_{k k}=V_{k}$ asymptotically, and the final electronic energy is $V_{1}$ or $V_{2}$ in TSH and DM trajectories whereas it is some value between $V_{1}$ and $V_{2}$ in Ehrenfest trajectories.) In the Ehrenfest calculations, the quenching probability was computed by the histogram method.

\section{RESULTS AND DISCUSSION}

The semiclassical trajectory calculations and the accurate quantum mechanical results are compared for the following six quantities $(i=1,2, \ldots, 6)$ :

$P_{\mathrm{R}} \quad$ the probability of reaction, which is the outcome in Eq. (29a)

$P_{\mathrm{Q}} \quad$ the probability of quenching, which is the outcome in Eq. (29b)

$P_{\mathrm{N}} \quad$ the total probability of a nonadiabatic event, which is the sum of $P_{\mathrm{R}}$ and $P_{\mathrm{Q}}$,

$F_{\mathrm{R}} \quad$ the reactive branching fraction, which is defined as $P_{\mathrm{R}} / P_{\mathrm{N}}$

$\left\langle E_{\text {int }}^{\prime}\right\rangle$ the average internal energy of the diatomic fragment in Eq. (29a)

$\left\langle E_{\text {int }}^{\prime \prime}\right\rangle$ the average internal energy of the diatomic fragment in Eq. (29b).

For the three probabilities the error $\varepsilon_{i \alpha}$ for quantity $i$ and test case $\alpha$ (nine cases for MXH and three case for YRH) is reported as the logarithmically averaged percentage error described elsewhere, ${ }^{31}$ and for the remaining three quantities, $F_{\mathrm{R}},\left\langle E_{\text {int }}^{\prime}\right\rangle$, and $\left\langle E_{\text {int }}^{\prime \prime}\right\rangle, \varepsilon_{i \alpha}$ the error is defined as the unsigned relative percentage error given by

$$
\varepsilon_{i \alpha}=\frac{\left|Q_{i \alpha}^{\text {traj }}-Q_{i \alpha}^{\text {quantal }}\right|}{Q_{i \alpha}^{\text {quantal }}} \times 100
$$


For case $\alpha$, the average error in probabilities is

$$
\bar{\varepsilon}_{a}(\operatorname{Prob})=\frac{1}{3} \sum_{i=1}^{3} \varepsilon_{i \alpha}
$$

and for the next three quantities, $F_{\mathrm{R}},\left\langle E_{\mathrm{int}}^{\prime}\right\rangle$, and $\left\langle E_{\text {int }}^{\prime \prime}\right\rangle$ (which represent how the energy of the system is fractionated into various nuclear coordinates), the average error is

$$
\bar{\varepsilon}_{\alpha}(\text { Fract })=\frac{1}{3} \sum_{i=4}^{6} \varepsilon_{i \alpha}
$$

These were then averaged over the nine MXH cases to give the MXH percentage error

$$
\operatorname{PE}(X ; \mathrm{MXH})=\frac{100}{9} \sum_{\alpha=1}^{9} \bar{\varepsilon}_{\alpha}(X),
$$

and similarly for the three YRH cases to give YRH percentage error

$$
\operatorname{PE}(X ; \mathrm{YRH})=\frac{100}{3} \sum_{\alpha=10}^{12} \bar{\varepsilon}_{\alpha}(X),
$$

where $X=1=$ "Prob" and $X=2=$ "Fract". Finally we averaged the two types of errors and two types of systems to obtain "average" mean unsigned percentage errors:

$$
\operatorname{PE}(\text { average })=\frac{1}{2} \sum_{X=1}^{2} \frac{\operatorname{PE}(X, \mathrm{MXH})+\operatorname{PE}(X, \mathrm{YRH})}{2} .
$$

We report numerical results for five quantities in Tables I and III: PE(Prob;MXH), PE(Fract;MXH), PE(Prob;YRH), PE(Fract;YRH) and PE(average). Semiclassical calculations were performed in the adiabatic (A), diabatic (D), and Calaveras County (CC) representations. ${ }^{1 \mathrm{~d}}$ The Calaveras County representation is defined as the representation with the fewest hopping attempts in a trajectory surface hopping calculation, and previous work has shown ${ }^{1 \mathrm{~d}}$ that this representation is, on average, the most accurate representation for trajectory surface hopping. 
Results for the SCDM, CSDM, and CSDM-C methods are presented in Table I for $E_{0}=0.1 E_{\mathrm{h}}$ and $C=1$ and using Eq. (28). The more coherent CSDM and CSDM-C methods are more accurate than the SCDM method for the probabilities in the YRH test cases when using the diabatic representation. For the rest of the test cases, the errors are similar for these three methods. This result indicates that for many systems the local coherence in the SCDM may be enough to obtain good results, but for certain cases a more coherent approach is necessary. In no case are the more coherent methods significantly less accurate than less coherent SCDM method.

A key reason for the greater success of the CSDM and CSDM-C methods appears to be that they are a little more coherent than SCDM. The MXH system has a diabatic crossing of the Landau-Zener-Teller (LZT) type so that in the strong interaction region, the decay-ofmixing time in the diabatic representation is greater than the decay-of-mixing time in the adiabatic representation, and thus the SCDM method in the diabatic representation works as well as the two new methods. On the other hand, the YRH system has a Rosen-ZenerDemkov (RZD) type of interaction so that in the strong interaction region both representations have similar decay-of-mixings, and this is apparently why the SCDM method does not work as well in the diabatic representation as the two new methods. The reason why the SCDM in the adiabatic representation is about as accurate as the CSDM and CSDM-C methods for both the MXH and YRH systems is apparently that the distribution of nonadiabatic coupling is much more localized than the diabatic coupling so that the DM trajectory on average has less deviation from the SE trajectory in the adiabatic representation than in the diabatic representation. In the other words, for a given system, the DM trajectory is more coherent in the adiabatic representation than in the diabatic representation. 
We used the anteater scheme to carry out ECP-TSH calculations and tested it for the nine MXH and three YRH cases mentioned above. The calculations were performed at various values of $\Omega_{\text {cutoff }}$ until convergence was reached. Two example cases in the adiabatic representation are presented in Table II where convergence with respect to $\Omega_{\text {cutoff }}$ is demonstrated. Table II shows that converged results are obtained for MXH SL $(1.1 / \mathrm{eV}, 0)$ with a relatively large $\Omega_{\text {cutoff }}$, but a relatively small $\Omega_{\text {cutoff }}$ is required for $\operatorname{YRH}(0.1)(1.1 / \mathrm{eV}, 0)$. Using the anteater scheme, one can simply set $\Omega_{\text {cutoff }}$ to zero. However, since previous workers did not always set $\Omega_{\text {cutoff }}=0$, Table II is included to demonstrate that for some cases, results may be sensitive to this parameter.

In Table III, errors are presented for the TFS- (where - denotes that the trajectory is reflected at frustrated hops $\left.{ }^{33,34}\right)$, TFS $+($ where + denotes that the trajectory is left unchanged at frustrated hops ${ }^{3,35}$ ), FSTUVV (where $\nabla \mathrm{V}$ denotes that the choice of whether to reflect or not at a frustrated hop is based on the gradient of the potential as discussed previously ${ }^{5}$ ), and $\mathrm{SE}^{6,9}$ methods. First, we will discuss the surface hopping methods (ECP-TSH, TFS+, TFS-, and FSTUVV). Table III shows that ECP-TSH method is less accurate on average than the TFS method for both the adiabatic and diabatic representations, in particular the branching probabilities are much worse in the ECP-TSH method than in the TFS method. This may be partly because some of frustrated hops in the ECP-TSH method are not frustrated in the TFS method as the TFS method allows hops to occur all along the trajectory. Note that for the YRH test cases, surface hopping methods in general give very different results in the adiabatic and diabatic representations. The FSTUVV method (which does not incorporate any explicit treatment 
of decoherence) is the most accurate surface hopping method, and it is the least dependent on the choice of electronic representation. It is possible that one could obtain better a surface hopping method by including an explicit treatment of decoherence in surface hopping using some but not all features of the ECP-TSH method, but we did not pursue this here.

Next we discuss the SCP methods (SE, SCDM, CSDM-C, and CSDM). The SE method is not accurate for real multidimensional systems. Table III shows that the CSDM, CSDM-C, and the SCDM methods are the most accurate with errors comparable to those ${ }^{32}$ for single-surface quasiclassical trajectory calculations. The CSDM and CSDM-C methods are less sensitive to the choice of electronic representation than the SCDM method. The CSDM method provides a simple and accurate solution to the problem of combining decay-of-mixing trajectories and coherent electronic state densities in non-Born-Oppenheimer dynamics.

To gain insight into the decay-of-mixing process incorporated into the successful decay-of-mixing algorithms, we computed the time average of the decay-of-mixing rates defined by Eq. (28). As the decay-of-mixing time is not meaningful in the initial and final legs of the trajectories where the coupling is essentially zero, we average only over the portions of the trajectories where $0.02 \leq \rho_{k k} \leq 0.98$. Recall that $\tau_{12}$ is the reciprocal of a first-order rate constant, and we should average rates, not their reciprocals. Therefore, to average the rates, we calculate the time average of $1 / \tau_{12}$ for this portion of each trajectory and then average these values over the ensemble of trajectories. Then, the result is re-expressed in terms of time units by taking a reciprocal: 


$$
\bar{\tau} \equiv \frac{1}{\left\langle 1 / \tau_{12}\right\rangle}
$$

The results for the three successful decay-of-mixing methods are shown in Table IV. We notice that the average decay-of-mixing is shorter in the adiabatic representation than in the diabatic representation for MXH (SB) with $j=2$ and $\mathrm{YRH}(0.2)$ with $j=0$, but the average decay-of-mixing is longer in the adiabatic representation than in the diabatic representation for MXH (WL) with $j=1$. The fact that CSDM does not require long algorithmic decay times to mimic quantum mechanics may be very important for understanding the physical origin of decoherence because requiring a large algorithmic component in the decay-of-mixing could mask the physical origin of decoherence.

We know for MXH (SB) with $j=2$ and YRH (0.2) with $j=0$ that the Calaveras County representation is the adiabatic representation, while for MXH (WL) with $j=1$, the Calaveras County representation is the diabatic representation. The examples in Table IV show that the representation with shorter average decay-of-mixing corresponds to the Calaveras County representation. This coincidence is also true for the other decay of mixing methods shown in Table II of Ref. 11. Thus it may be unnecessary to run surface hopping calculations to determine the Calaveras County representation; one can simply use the representation in which $\bar{\tau}$ is shorter.

Table IV also shows the average number of local minima per trajectory of Eq. (20) for the CSDM method and of Eq. (21) for the CSDM-C method. These statistics are important because it is at such local minima where the electronic density matrix for computing the switching probability is set equal to the decay of mixing electronic density matrix. Some readers might be surprised at how large these numbers are since one often thinks of polyatomic collisions as similar to atomic collisions, where this number might often 
be only 2. For the present cases the number of local minima in the CSDM-C method is roughly equal to 2.5 times the number of local minima in the CSDM method. This is because the CSDM-C method often has a local minimum at a vibrational turning point, which is not a local minimum for the CSDM method.

The present study illustrates the importance of properly balancing coherence and decoherence, and the formulation in Eq. (28) is reasonable for studying collisions and photochemical processes in small as well as big molecules. Nevertheless a deeper understanding of the best ways to incorporate coherence and decoherence into semiclassical theory would be worthwhile.

Although the present article only involves two-state applications, the new methods (FSTU and decay of mixing) that we have presented are all defined for the general multistate case.

\section{CONCLUDING REMARKS}

Recent interest in quantum measurement theory has led to increased interest in decoherence and its relation to the classical limit of quantum mechanics. ${ }^{36-38}$ In a more specific context, Thachuk et al. ${ }^{12}$ illuminated the key roles of coherence and decoherence for two-state one-dimensional system interacting with an oscillatory time-dependent electric field, and Rossky and coworkers ${ }^{10}$ emphasized the importance of decoherence in the simulation of electronically nonadiabatic processes in the condensed phases. Hack and one of the authors ${ }^{23}$ developed a formalism, called natural decay of mixing, for adding decoherence to the semiclassical Ehrenfest method and showed that the resulting treatment were the most accurate of all available semiclassical trajectory methods for non-Born-Oppenheimer 
collisions. We showed in a previous paper ${ }^{11}$ that we obtain more accurate results for electronically nonadiabatic collisions by adding more coherence to the natural decay of mixing algorithm; the resulting algorithm was called self-consistent decay of mixing (SCDM). In the present paper, by using a coherent complete passage of each strong interaction region to compute the local switching probability of the SCDM method, we have further improved the decay of mixing method for nonadiabatic dynamics, resulting in a new algorithm called coherent switches with decay of mixing (CSDM). We also consider the exact complete passage (ECP) algorithm of Parlant and Gislason ${ }^{14}$ for adding coherence to trajectory surface hopping (TSH) calculations. Although the ECP-TSH method does not improve the TFS method on average, the key feature of this method is borrowed for the SCDM method, where it leads to the CSDM method.

It is important to emphasize that the new decay-of-mixing method, CSDM, performs almost equally well in the adiabatic and diabatic representations. The reasons why this is important can be summarized as follows: First, it is not always possible to predict (when the accurate quantal results are unavailable) which representation is preferred. Second and even more significant, for complex systems there can be regions of configuration space in which the adiabatic representation is preferred, but, for the same system with the same initial conditions, there can be other regions of configuration space where the diabatic representation is more natural. In particular, in real molecular dynamics simulations, one may encounter systems with several qualitatively different kinds of coupling regions. A good example would be a system with a conical intersection where close to the cone, one has an LZT-type of avoided crossing like MXH, but in regions far from the cone one has RZD-type interactions as in YRH. Another example 
would be a three-state system where the coupling between states 1 and 2 is most naturally treated in the adiabatic representation but the couplings between states 1 and 3 or 2 and 3 are more diabatic. For such a system, one needs a method that works equally well in both representations. CSDM is especially well suited to satisfy this need for multidimensional dynamics.

One will be much safer to treat general complex systems if one has method that yields accurate results in both representations, and CSDM can fill this need.

The key results of this paper are in Table III. This table shows that for twelve test cases involving five different atom-diatom systems, the CSDM method is the most accurate self-consistent potential (SCP) method, and it is also more accurate than the FSTU " V method, which we have found to be is the most accurate TSH method. As compared to surface hopping methods, the CSDM method has the advantage that coordinates and momenta are continuous with continuous derivatives along each trajectory; and there are no frustrated hops. As compared to the semiclassical Ehrenfest method, it contains a better treatment of decoherence. The computational effort for practical problems is nearly the same as for TSH and semiclassical Ehrenfest methods. The CSDM method is suited for general polyatomic applications.

\section{ACKNOWLEDGMENTS}

The authors are grateful to Dmitri Babikov for a helpful conversation. This work was supported in part by the National Science Foundation under grant no. CHE00-92019. 


\section{APPENDIX A: SWITCHING PROBABILITY FOR MULTISTATE CASES}

We can formulate the switching probability for the mulitstate case by following Tully's fewest switching method, ${ }^{3}$ and the switching probability from the current decoherent state $K$ to another decoherent state $K^{\prime}$ is given by

$$
P_{K \rightarrow K^{\prime}}=\max \left(\frac{\left(b_{K^{\prime} K}-\dot{\rho}_{K K}^{D}\right) d t}{\rho_{K K}}, 0\right)
$$

where we have

$$
b_{K^{\prime} K}=-2 \hbar^{-1} \operatorname{Im}\left(\rho_{K^{\prime} K} U_{K^{\prime} K}\right)
$$

for the diabatic representation where $U_{K^{\prime} K}=U_{K K^{\prime}}$, and

$$
b_{K^{\prime} K}=2 \operatorname{Re}\left(\rho_{K^{\prime} K} \dot{\mathbf{R}} \cdot \mathbf{d}_{K K^{\prime}}\right)
$$

for the adiabatic representation where $\mathbf{d}_{K^{\prime} K}=-\mathbf{d}_{K K^{\prime}}$. (Recall that $\rho_{K K^{\prime}}=\rho_{K^{\prime} K}^{*}$.) The NDM method requires that both $\rho_{K K}$ and $\rho_{K^{\prime} K}$ in Eq. (A1)-(A3) are calculated from the decay of mixing electronic density of Eq. (9). The SCDM and CSDM methods require omitting $\dot{\rho}_{K K}^{D}$ in Eq. (A1); furthermore in the CSDM method both $\rho_{K K}$ and $\rho_{K^{\prime} K}$ should also be replaced by $\widetilde{\rho}_{K K}$ and $\widetilde{\rho}_{K^{\prime} K}$ defined in Sec. IV. C.

It is useful to point out the relationship to the coherent term in Eq. (9), i.e.,

$$
\dot{\rho}_{K K}^{C}=\sum_{K^{\prime} \neq K} b_{K K^{\prime}}
$$

\section{APPENDIX B: RESULTS WITH SELECTED $E_{0}$ VALUES}

In Table B-I results for $E_{0}=0.05,0.1$, and $0.2 E_{\mathrm{h}}$ are presented for the MXH system using the SCDM, CSDM, and CSDM-C methods. The overall percentage errors in the last 
column of the table show that the results are relatively insensitive to the value of $E_{0}$ and are slightly more accurate for $E_{0}=0.1 E_{\mathrm{h}}$. A similar conclusion is drawn from the results in Table B-II for the YRH system. 


\section{REFERENCES}

1. For reviews that compare methods, see: (a) S. Chapman, Adv. Chem. Phys. 82, 423 (1992). (b) J. C. Tully, in Modern Methods for Multidimensional Dynamics Computations in Chemistry, edited by D. L. Thompson (World Scientific, Singapore, 1998), p. 34. (c) J. C. Tully, Faraday Discussions 110, 407 (1998). (d) M. D. Hack and D. G. Truhlar, J. Phys. Chem. A 104, 7917 (2000). (e) A. W. Jasper, B. K. Kendrick, C. A. Mead, and D. G. Truhlar, in Modern Trends in Chemical Reaction Dynamics, edited by X. Yang and K. Liu (World Scientific, Singapore, 2004), pp. 329-392. (f) A. W. Jasper, C. Zhu, S. Nangia, and D. G. Truhlar, Faraday Discussions 127, 1 (2004).

2. W. H. Miller and T. F. George, J. Chem. Phys. 56, 5637 (1972).

3. J. C. Tully, J. Chem. Phys. 93, 1061 (1990).

4. A. W. Jasper, S. N. Stechmann, and D. G. Truhlar, J. Chem. Phys. 116, 5424 (2002), 117, 10247(E) (2002).

5. A. W. Jasper and D. G. Truhlar, Chem. Phys. Lett. 369, 60 (2003).

6. H.-D. Meyer and W. H. Miller, J. Chem. Phys. 70, 3214 (1979).

7. (a) D. A. Micha, J. Chem. Phys. 78, 7138 (1983). (b) M. Amarouche, F. X. Gadea, and J. Durup, Chem. Phys. 130, 145 (1989).

8. A. Garcia-Vela, R. B. Gerber, and D. G. Imre, J. Chem. Phys. 97, 7242 (1992).

9. M. D. Hack, A. W. Jasper, Y. L. Volobuev, D. W. Schwenke, and D. G. Truhlar, J. Phys. Chem. A 104, 217 (2000).

10. (a) O. V. Prezhdo and P. J. Rossky, J. Chem. Phys. 107, 5863 (1997). (b,c) K. F. Wong and P. J. Rossky, J. Chem. Phys. 116, 8418, 8429 (2002). 
11. C. Zhu, A. W. Jasper, and D. G. Truhlar, J. Chem. Phys. 120, 5543 (2004).

12. M. Thachuk, M. Y. Ivanov, and D. M. Wardlaw, J. Chem. Phys. 109, 5747 (1998).

13. G. Parlant and E. A. Gislason, J. Chem. Phys. 91, 4416 (1989).

14. (a) G. Parlant and M. H. Alexander, J. Chem. Phys. 92, 2287 (1990). (b) M. Sizun, J.B. Song, and E. A. Gislason, J. Chem. Phys. 109, 4815 (1998). (c) D. Babikov, E. A. Gislason, M. Sizun, F. Aguillon, and V. Sidis, J. Chem. Phys. 112, 7032 (2000).

15. P. J. Kuntz, J. Kendrick, and W. N. Whitton, Chem. Phys. 38, 147 (1979).

16. N. C. Blais and D. G. Truhlar, J. Chem. Phys. 79, 1334 (1983).

17. (a) C. C. Martens and J. Y. Fang, J. Chem. Phys. 106, 4918 (1997). (b) A. Donoso and C. C. Martens, J. Phys. Chem. A 102, 4291 (1998). (c) A. Donoso and C. C. Martens, J. Chem. Phys. 112, 3980 (2000). (d) A. Donoso and C. C. Martens, Int. J. Quantum Chem. 90, 1348 (2002).

18. See for example, (a) R. Kapral and G. Ciccotti, J. Chem. Phys. 110, 8919 (1999), (b) M. Sauter, U. Manthe, and G. Stock, J. Chem. Phys. 114, 2001 (2001).

19. (a) T. J. Martinez, M. Ben-Nun, and R. D. Levine, J. Phys. Chem. A 101, 6389 (1997). (b) M. D. Hack, A. M. Wensmann, D. G. Truhlar, M. Ben-Nun, and T. J. Martinez, J. Chem. Phys. 115, 1172 (2001).

20. (a) C. A. Mead and D. G. Truhlar, J. Chem. Phys. 77, 6090 (1982). (b) B. K. Kendrick, C. A. Mead, and D. G. Truhlar, Chem. Phys. 277, 311 (2002).

21. H.-D. Meyer and W. H. Miller, J. Chem. Phys. 72, 2272 (1980).

22. M. D. Hack, A. W. Jasper, Y. L. Volobuev, D. W. Schwenke, and D. G. Truhlar, J. Phys. Chem. A 103, 6309 (1999). 
23. M. D. Hack and D. G. Truhlar, J. Chem. Phys. 114, 9305 (2001).

24. (a) P. J. Kuntz, J. Chem. Phys. 95, 141 (1991). (b) P. J. Kuntz and J. J. Hogreve, J. Chem. Phys. 95, 156 (1991). (c) P. J. Kuntz, I. Paidarová, and R. Polák, Chem. Phys. 209, 389 (1996). (d) W. Jakubetz, J. N. L. Connor, and P. J. Kuntz, Phys. Chem. Chem. Phys. 1, 1213 (1999).

25. L. Turi and P. J. Rossky, J. Chem. Phys. 120, 3688 (2004).

26. G. A. Fiete and E. J. Heller, Phys. Rev. A 68, 22112 (2003).

27. Y. L. Volobuev, M. D. Hack, M. S. Topaler, and D. G. Truhlar, J. Chem. Phys. 112, $9716(2000)$.

28. A. W. Jasper, M. D. Hack, and D. G. Truhlar, J. Chem. Phys. 115, 1804 (2001).

29. W. H. Press, S. A. Teukolsky, W. T. Vetterling, and B. P. Flannery, Numerical Recipes in FORTRAN, $2^{\text {nd }}$ ed. (Cambridge University Press, Cambridge, UK 1994); pp. 716-725.

30. M. S. Topaler, M. D. Hack, T. C. Allison, Y.-P. Liu, S. L. Mielke, D. W. Schwenke, and D. G. Truhlar, J. Chem. Phys. 106, 8699 (1997).

31. T. C. Allison and D. G. Truhlar, in Modern Methods for Multidimensional Dynamics Computations in Chemistry, edited by D. L. Thompson (World Scientific, Singapore, 1998), pp. 618-712.

32. M. S. Topaler, T. C. Allison, D. W. Schwenke, and D. G. Truhlar, J. Chem. Phys. 109, 3321 (1998), 113, 3928(E) (2000).

33. N. C. Blais and D. G. Truhlar, J. Chem. Phys. 79, 1334 (1983).

34. S. Hammes-Schiffer and J. C. Tully, J. Chem. Phys. 101, 4657 (1994).

35. U. Müller and G. Stock, J. Chem. Phys. 107, 6230 (1997). 
36. R. B. Griffiths, Consistent Quantum Theory (Cambridge University Press, Cambridge, UK, 2002).

37. J. P. Paz and W. H. Zurek, Phys. Rev. Lett. 82, 5181 (1999).

38. R. Blume-Kohout and W. H. Zurek, Phys. Rev. A 61, 032104 (2003). 
Table I. Average percentage errors in the probabilities and internal energy distributions for nine MXH and three YRH test cases

\begin{tabular}{|c|c|c|c|c|c|c|}
\hline \multirow{2}{*}{ Method } & \multirow{2}{*}{ Rep. } & \multicolumn{2}{|c|}{ MXH } & \multicolumn{2}{|c|}{ YRH } & \multirow{2}{*}{ Average } \\
\hline & & Prob & Fract & Prob & Fract & \\
\hline \multirow[t]{2}{*}{$\mathrm{CSDM}^{\mathrm{a}}$} & A & 26 & 21 & 21 & 18 & 21 \\
\hline & $\mathrm{D}$ & 29 & 20 & 41 & 22 & 28 \\
\hline \multirow[t]{2}{*}{ CSDM-Ca } & A & 26 & 20 & 20 & 17 & 21 \\
\hline & $\mathrm{D}$ & 28 & 20 & 49 & 22 & 30 \\
\hline \multirow[t]{2}{*}{$\mathrm{SCDM}^{\mathrm{a}}$} & A & 27 & 22 & 20 & 17 & 21 \\
\hline & D & 28 & 20 & 69 & 22 & 35 \\
\hline
\end{tabular}

abtained using $E_{0}=0.1 \mathrm{E}_{\mathrm{h}}$ and $C=1$ in Eq. (28). 
Table II. Convergence of mean unsigned percentage errors (\%) in the branching probabilities and final internal energies for the ECP-TSH method in the adiabatic representation.

\begin{tabular}{|c|c|c|c|c|c|c|c|c|c|}
\hline$\Omega_{\text {cutoff }}(\mathrm{eV})$ & $P_{\mathrm{R}}$ & $P_{\mathrm{Q}}$ & $P_{\mathrm{N}}$ & $F_{\mathrm{R}}$ & $E_{\text {int }}^{\prime}$ & $E_{\mathrm{int}}^{\prime \prime}$ & Prob & Fract & All \\
\hline \multicolumn{10}{|c|}{$\operatorname{MXH~SL~}(E=1.1 \mathrm{eV}, j=0)$} \\
\hline $6 \times 10^{-1}$ & 100 & 516 & 44 & 188 & 2 & 12 & 220 & 67 & 144 \\
\hline $6 \times 10^{-2}$ & 240 & 141 & 28 & 165 & 2 & 4 & 136 & 57 & 97 \\
\hline $6 \times 10^{-3}$ & 230 & 120 & 28 & 158 & 2 & 5 & 126 & 55 & 90 \\
\hline $6 \times 10^{-4}$ & 220 & 102 & 28 & 150 & 2 & 3 & 117 & 52 & 84 \\
\hline \multicolumn{10}{|c|}{$\mathrm{YRH}(0.1)(E=1.1 \mathrm{eV}, j=0)$} \\
\hline $6 \times 10^{-5}$ & 273 & 282 & 281 & 2 & 3 & 4 & 279 & 3 & 141 \\
\hline $6 \times 10^{-7}$ & 264 & 279 & 276 & 3 & 2 & 4 & 273 & 3 & 138 \\
\hline
\end{tabular}


Table III. Mean unsigned relative errors (\%) in the branching probabilities and internal energy distributions for nine MXH and three YRH test cases

\begin{tabular}{|c|c|c|c|c|c|c|}
\hline \multirow{2}{*}{ Method } & \multirow{2}{*}{ Rep. } & \multicolumn{2}{|c|}{ MXH } & \multicolumn{2}{|c|}{ YRH } & \multirow{2}{*}{ Average } \\
\hline & & Prob & Fract & Prob & Fract & \\
\hline \multicolumn{7}{|c|}{ Trajectory surface hopping methods } \\
\hline \multirow{3}{*}{ ECP-TSH } & A & 90 & 47 & 377 & 4 & 130 \\
\hline & $\mathrm{D}$ & 123 & 47 & 1016 & 30 & 304 \\
\hline & $\mathrm{CC}$ & 118 & 48 & 377 & 4 & 137 \\
\hline \multirow[t]{3}{*}{ TFS+ } & A & 57 & 34 & 53 & 18 & 41 \\
\hline & $\mathrm{D}$ & 54 & 26 & 723 & 49 & 213 \\
\hline & $\mathrm{CC}$ & 59 & 32 & 53 & 18 & 41 \\
\hline \multirow[t]{3}{*}{ TFS- } & A & 54 & 29 & 43 & 15 & 35 \\
\hline & $\mathrm{D}$ & 47 & 22 & 548 & 29 & 161 \\
\hline & $\mathrm{CC}$ & 50 & 28 & 43 & 15 & 34 \\
\hline \multirow{3}{*}{ FSTUVV } & A & 52 & 30 & 31 & 19 & 33 \\
\hline & $\mathrm{D}$ & 45 & 20 & 230 & 26 & 80 \\
\hline & $\mathrm{CC}$ & 45 & 28 & 31 & 19 & 33 \\
\hline \multicolumn{7}{|c|}{ Self-consistent potential methods } \\
\hline SE & all & 132 & 40 & $\mathrm{a}$ & $\mathrm{a}$ & - \\
\hline \multirow[t]{3}{*}{ SCDM } & A & 27 & 22 & 20 & 17 & 21 \\
\hline & $\mathrm{D}$ & 28 & 20 & 69 & 22 & 35 \\
\hline & $\mathrm{CC}$ & 30 & 21 & 20 & 17 & 22 \\
\hline \multirow[t]{3}{*}{ CSDM-C } & A & 26 & 20 & 20 & 17 & 21 \\
\hline & $\mathrm{D}$ & 28 & 20 & 49 & 22 & 30 \\
\hline & $\mathrm{CC}$ & 28 & 20 & 20 & 17 & 21 \\
\hline \multirow[t]{3}{*}{ CSDM } & A & 26 & 21 & 21 & 18 & 21 \\
\hline & $\mathrm{D}$ & 29 & 20 & 41 & 22 & 28 \\
\hline & $\mathrm{CC}$ & 28 & 21 & 21 & 18 & 22 \\
\hline
\end{tabular}

aNone of the trajectories finished in product arrangements. 
Table IV. Mean decay-of-mixings (fs) for SCDM, CSDM and CSDM-C

\begin{tabular}{ccccc}
\hline Method & Rep. & $\begin{array}{c}\text { MXH SB } \\
j=2\end{array}$ & $\begin{array}{c}\text { MXH WL } \\
j=1\end{array}$ & $\begin{array}{c}\text { YRH } 0.2 \\
1.02 \mathrm{eV}, j=0\end{array}$ \\
\hline \multirow{2}{*}{ SCDM } & A & 5.6 & 5.3 & 33. \\
& D & 6.6 & 4.9 & 36. \\
& & & & \\
CSDM-C & A & $5.7(231)^{\mathrm{a}}$ & $6.0(118)$ & $33(195)$ \\
& $\mathrm{D}$ & $6.5(237)$ & $4.9(117)$ & $36(195)$ \\
& & & & \\
CSDM & $\mathrm{A}$ & $7.8(99)$ & $8.6(59)$ & $36(78)$ \\
& $\mathrm{D}$ & $9.7(98)$ & $7.6(58)$ & $37(78)$ \\
\hline
\end{tabular}

aNumbers in parentheses are the average number of minima per trajectory of Eq. (25) for the CSDM method and of Eq. (26) for the CSDM-C method. As in Table I and III, we used $C=1$ and $E_{0}=0.1 \mathrm{E}_{\mathrm{h}}$ in Eq. (28). 
Table B-I. A detailed comparison of results for selected $E_{0}$ values using CSDM, CSDM-C, and SCDM methods for the MXH system.

\begin{tabular}{|c|c|c|c|c|c|c|c|c|c|c|c|}
\hline Method & $E_{0}$ & Rep. & $P_{\mathrm{R}}$ & $P_{\mathrm{Q}}$ & $P_{\mathrm{N}}$ & $F_{\mathrm{R}}$ & $E_{\mathrm{int}}^{\prime}$ & $E_{\mathrm{int}}^{\prime \prime}$ & Prob & Fract & All \\
\hline \multirow[t]{9}{*}{ CSDM } & 0.05 & $\mathrm{~A}$ & 38 & 34 & 24 & 20 & 23 & 25 & 32 & 23 & 27 \\
\hline & & $\mathrm{D}$ & 23 & 46 & 21 & 21 & 16 & 20 & 30 & 19 & 24 \\
\hline & & $\mathrm{CC}$ & 36 & 43 & 25 & 22 & 22 & 24 & 35 & 22 & 29 \\
\hline & 0.1 & A & 24 & 35 & 18 & 17 & 23 & 21 & 26 & 21 & 23 \\
\hline & & $\mathrm{D}$ & 29 & 43 & 15 & 25 & 18 & 18 & 29 & 20 & 25 \\
\hline & & $\mathrm{CC}$ & 24 & 42 & 18 & 19 & 23 & 21 & 28 & 21 & 24 \\
\hline & 0.2 & A & 43 & 38 & 14 & 31 & 27 & 18 & 32 & 25 & 28 \\
\hline & & $\mathrm{D}$ & 60 & 42 & 12 & 36 & 20 & 15 & 38 & 24 & 31 \\
\hline & & $\mathrm{CC}$ & 43 & 41 & 14 & 32 & 26 & 17 & 33 & 25 & 29 \\
\hline \multirow[t]{9}{*}{ CSDM-C } & 0.05 & A & 36 & 35 & 24 & 19 & 23 & 24 & 32 & 22 & 27 \\
\hline & & $\mathrm{D}$ & 23 & 46 & 22 & 21 & 16 & 20 & 30 & 19 & 24 \\
\hline & & $\mathrm{CC}$ & 35 & 42 & 25 & 20 & 22 & 24 & 34 & 22 & 28 \\
\hline & 0.1 & A & 25 & 34 & 18 & 17 & 23 & 21 & 26 & 20 & 23 \\
\hline & & $\mathrm{D}$ & 27 & 43 & 16 & 24 & 20 & 18 & 28 & 20 & 24 \\
\hline & & $\mathrm{CC}$ & 25 & 41 & 19 & 18 & 23 & 21 & 28 & 20 & 24 \\
\hline & 0.2 & A & 37 & 37 & 14 & 28 & 26 & 18 & 29 & 24 & 27 \\
\hline & & $\mathrm{D}$ & 63 & 43 & 11 & 37 & 21 & 15 & 39 & 24 & 32 \\
\hline & & $\mathrm{CC}$ & 38 & 42 & 14 & 29 & 25 & 18 & 31 & 24 & 28 \\
\hline \multirow[t]{9}{*}{ SCDM } & 0.05 & A & 40 & 35 & 23 & 22 & 22 & 25 & 33 & 23 & 28 \\
\hline & & $\mathrm{D}$ & 24 & 44 & 22 & 19 & 18 & 20 & 30 & 19 & 25 \\
\hline & & $\mathrm{CC}$ & 36 & 44 & 25 & 21 & 22 & 24 & 35 & 22 & 29 \\
\hline & 0.1 & A & 26 & 38 & 18 & 20 & 23 & 22 & 27 & 22 & 25 \\
\hline & & $\mathrm{D}$ & 24 & 44 & 17 & 23 & 20 & 18 & 28 & 20 & 24 \\
\hline & & $\mathrm{CC}$ & 26 & 45 & 19 & 21 & 23 & 21 & 30 & 21 & 26 \\
\hline & 0.2 & A & 39 & 34 & 15 & 28 & 27 & 19 & 30 & 24 & 27 \\
\hline & & $\mathrm{D}$ & 62 & 45 & 12 & 37 & 22 & 14 & 40 & 25 & 32 \\
\hline & & $\mathrm{CC}$ & 40 & 43 & 15 & 30 & 27 & 17 & 33 & 25 & 29 \\
\hline
\end{tabular}


Table B-II. A detailed comparison of results for selected $E_{0}$ values using CSDM, CSDM-C, and SCDM methods for the YRH system.

\begin{tabular}{|c|c|c|c|c|c|c|c|c|c|c|c|}
\hline Method & $E_{0}$ & Rep. & $P_{\mathrm{R}}$ & $P_{\mathrm{Q}}$ & $P_{\mathrm{N}}$ & $F_{\mathrm{R}}$ & $E_{\text {int }}^{\prime}$ & $E_{\text {int }}^{\prime \prime}$ & Prob & Fract & All \\
\hline \multirow[t]{9}{*}{ CSDM } & 0.05 & A & 31 & 26 & 23 & 28 & 9 & 22 & 27 & 20 & 23 \\
\hline & & $\mathrm{D}$ & 30 & 74 & 66 & 28 & 6 & 20 & 57 & 18 & 37 \\
\hline & & $\mathrm{CC}$ & 31 & 26 & 23 & 28 & 9 & 22 & 27 & 20 & 23 \\
\hline & 0.1 & A & 16 & 28 & 19 & 28 & 7 & 20 & 21 & 18 & 20 \\
\hline & & $\mathrm{D}$ & 41 & 47 & 36 & 39 & 5 & 22 & 41 & 22 & 32 \\
\hline & & $\mathrm{CC}$ & 16 & 28 & 19 & 28 & 7 & 20 & 21 & 18 & 20 \\
\hline & 0.2 & A & 35 & 30 & 20 & 36 & 7 & 19 & 28 & 20 & 24 \\
\hline & & $\mathrm{D}$ & 76 & 28 & 21 & 47 & 6 & 23 & 42 & 25 & 34 \\
\hline & & $\mathrm{CC}$ & 35 & 30 & 20 & 36 & 7 & 19 & 28 & 20 & 24 \\
\hline \multirow[t]{9}{*}{ CSDM-C } & 0.05 & A & 31 & 26 & 23 & 28 & 9 & 22 & 27 & 20 & 23 \\
\hline & & $\mathrm{D}$ & 60 & 82 & 75 & 35 & 6 & 20 & 72 & 20 & 46 \\
\hline & & $\mathrm{CC}$ & 31 & 26 & 23 & 28 & 9 & 22 & 27 & 20 & 23 \\
\hline & 0.1 & A & 16 & 26 & 18 & 23 & 6 & 20 & 20 & 17 & 18 \\
\hline & & $\mathrm{D}$ & 47 & 56 & 44 & 39 & 6 & 22 & 49 & 22 & 36 \\
\hline & & $\mathrm{CC}$ & 16 & 26 & 18 & 23 & 6 & 20 & 20 & 17 & 18 \\
\hline & 0.2 & A & 34 & 28 & 19 & 36 & 6 & 19 & 27 & 20 & 24 \\
\hline & & $\mathrm{D}$ & 68 & 34 & 22 & 48 & 7 & 23 & 41 & 26 & 34 \\
\hline & & $\mathrm{CC}$ & 34 & 28 & 19 & 36 & 6 & 19 & 27 & 20 & 24 \\
\hline \multirow[t]{9}{*}{ SCDM } & 0.05 & A & 33 & 26 & 23 & 27 & 9 & 21 & 28 & 19 & 23 \\
\hline & & $\mathrm{D}$ & 94 & 121 & 113 & 36 & 7 & 21 & 109 & 21 & 65 \\
\hline & & $\mathrm{CC}$ & 33 & 26 & 23 & 27 & 9 & 21 & 28 & 19 & 23 \\
\hline & 0.1 & A & 18 & 26 & 18 & 24 & 7 & 19 & 20 & 17 & 19 \\
\hline & & $\mathrm{D}$ & 51 & 83 & 72 & 36 & 7 & 23 & 69 & 22 & 45 \\
\hline & & $\mathrm{CC}$ & 18 & 26 & 18 & 24 & 7 & 19 & 20 & 17 & 19 \\
\hline & 0.2 & A & 30 & 27 & 18 & 35 & 7 & 18 & 25 & 20 & 22 \\
\hline & & $\mathrm{D}$ & 49 & 50 & 36 & 48 & 6 & 24 & 45 & 26 & 36 \\
\hline & & $\mathrm{CC}$ & 30 & 27 & 18 & 35 & 7 & 18 & 25 & 20 & 22 \\
\hline
\end{tabular}

